\title{
Genetik psychischer Erkrankungen
}

$\mathrm{D}$ ie Artikel des vorliegenden Heftes sollen dem Leser einen Einblick in den Stand der genetischen Forschung bei psychiatrischen Erkrankungen vermitteln. Den hierfür ausgewählten Krankheiten gemeinsam ist ihre große Relevanz für die Allgemeinheit: Angstund Suchterkrankungen gehören mit Ein-Jahres-Prävalenzraten von 12 bzw. 7,5\% zu den am häufigsten auftretenden psychischen Störungen. Schizophrenien und bipolar depressive Störungen nehmen den fünften und sechsten Platz auf der WHO-Liste der „Global Burden of Disease" ein, und die Alzheimersche Erkrankung wird nicht zuletzt aufgrund der demographischen Entwicklung in den Industriestaaten ein immer wichtigeres Thema der Gesundheitspolitik.

Eine weitere Gemeinsamkeit der dargestellten Erkrankungen ist, dass an der Entstehung genetische Faktoren beteiligt sind. Die Aufklärung dieser genetischen Grundlagen auf der molekularen Ebene wird zur Entwicklung neuer, rational begründeter und individualisierter Medikamente beitragen. Darüber hinaus liegt in der Identifizierung von Gen-Umweltinteraktionen ein gewaltiges Potenzial zur Entwicklung von nicht medikamentösen Interventionen zur Prophylaxe und Therapie. Sind die unterschiedlichen Interaktionsprofile bekannt, dann können entsprechende Verhaltensmaßnahmen ergriffen werden. Diese können zum Beispiel allgemeiner Natur sein, wie die Stressreduktion, oder sehr spezielle Maßnahmen umfassen, wie die Psychotherapie.

Die Fortschritte auf dem Gebiet der Genetik sind enorm. Deutschland hat seine Forschung mit der Beteiligung am Humanen Genomforschungsprojekt (seit 1994) und den im Jahre 2001 initiierten Nationalen Genomforschungsnetzen verstärkt. Bis zur Entschlüsselung aller krankheitsrelevanten Gene und der Aufklärung ihrer komplexen Wirkzusammenhänge ist es aber noch ein weiter Weg. Gerade wenn heute schon vielfach die „Postgenomische Ära" heraufbeschworen wird, ist dies eher als Ausdruck überschwänglicher Hoffnungen zu sehen, als dass es die Realität widerspiegelt. Hier muss man vorsichtig sein, um unrealistische Hoffnungen und Befürchtungen der Bevölkerung nicht noch zu verstärken. Im Allgemeinen hat die Bevölkerung eine positive, wenn auch kritische Einstellung gegenüber dieser Forschung. Dies ist eine gute Voraussetzung für eine offene Auseinandersetzung mit den im Rahmen der molekulargenetischen Forschung auftretenden ethischen Konflikten bzw. Fragestellungen.
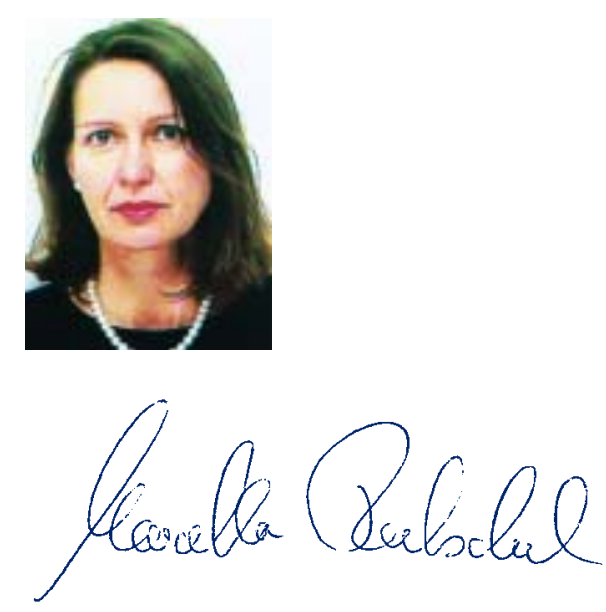

Prof. Dr. Marcella Rietschel, Mannheim 